\title{
Generative Networks and Royalty-Free Products
}

\author{
iD Yasin Özkan ${ }^{1}$, (DPakize Erdoğmuş² \\ ${ }^{1}$ Corresponding Author; Zonguldak Bulent Ecevit University, Vocational School, Department of \\ Computer Technology Program; yasin.ozkan@beun.edu.tr; +903723191950 \\ ${ }^{2}$ Duzce University, Faculty of Engineering, Department of Computer Engineering; \\ pakizeerdogmus@duzce.edu.tr; +903805421036
}

Received 21 April 2020; Revised 6 September 2020; Accepted 8 December 2020; Published online 30 December 2020

\begin{abstract}
In recent years, with the increasing power of computers and Graphics Processing Units (GPUs), vast variety of deep neural networks architectures have been created and realized. One of the most interesting and generative type of the networks are Generative Adversarial Networks (GANs). GANs are used to create things such as music, images or a film scenerio. GANs consist of two networks working simultaneously. Generative network captures data distribution and discriminative network estimates the probability of the Generative Network output, coming from training data of discriminative network. The objective is to both maximizing the generative network products reality and minimize the discriminative network classification error. This procedure is a minimax two-player game. In this paper, it has been aimed to review the latest studies with GANs, to gather the recent studies in an article and to discuss the possible issues with royalty free products created by GANs. With this aim, from 2018 to today, the studies on GANs have been gathered to the citation numbers. As a result, the recent studies with GANs have been summarized and the potential issues related to GANs have been submitted.
\end{abstract}

Keywords: deep learning, royalty-free products, authentication

\section{Üretken Ağlar ve Telifsiz Ürünler}

\section{Öz}

Son yıllarda, bilgisayarların ve Grafik İşlem Birimlerinin (GPU'ların) artan gücüyle, çok çeşitli derin sinir ağları mimarileri oluşturulmuş ve gerçekleştirilmiştir. En ilginç ve üretken ağ türlerinden biri de Üretken Çekişmeli Ağlardır (GANs). GAN ağları müzik, görüntü ve film senaryolarının üretiminde kullanılmaktadır. GAN ağları eş zamanlı çalışan iki ağ yapısından oluşmaktadır. Üretici ağ, veri dağıtımını üstlenmekte ayırıcı ağlar ise, ayrımcı ağın veya üretken ağ ürününün eğitim verilerinden gelen Üretken A ̆g çıktısının olasılığını tahmin etmektedir. Amaç hem üretken ağın ürettiği verinin gerçekliğini maksimize ederken, ayırıcı ağın da hatasını minimize etmektir. Bu süreç iki oyunculu bir minimax problemidir.

$\mathrm{Bu}$ çalışmada GAN ağları ile ilgili yapılan son çalışmaların gözden geçirilerek bir makale altında bir araya getirilmesi ve GAN ağları ile üretilen telifsiz ürünler ile ortaya çıkacak olası konuların tartışılması amaçlanmıştır. Bu amaç ile 2018'den günümüze bu konuda yapılmış olan çalışmaların atıf sayısı en yüksek olanları bu çalışmada bir araya getirilmiştir.

Sonuç olarak GAN ağları ile yapılmış bu çalışmaların özet ve sonuçları bir tablo haline getirilerek sunulmaktadır. Bu şekilde GAN ağlarının mevcut uygulamaları bu çalışmada ortaya konulmaktadır. Yine GAN ağları ile ilgili olası sorunların ne olacağı da bu çalışmada sunulmaktadır.

Anahtar Kelimeler: derin öğrenme, telifsiz ürünler, kimlik doğrulama

\section{Introduction}

With the industry revolution, mankind became acquainted with the machines working instead of them. Since the first industry machines have been used for workforce, they can be accepted as blue collar workers. With booming technology during the Second World War, mankind met a very brilliant machine called computer. Computers have been managing the industrial machines, making calculations for an accountant, or increasing the productivity using Operations Research methods or 
algorithms. So the second types of machines have been accepted as white-collar workers. Even if they are very good at computing and analyzing, they can't be competing with the peoples on recognition and learning from patterns or samples. The researchers have been working on more brilliant computers, which isusing "Artificial intelligence” This type of computers are not only computing, analyzing and saving but also producing art and music, recognizing the peoples and driving car.

Artificial intelligence, coined by John McCarty, has been introduced in the Dartmouth Conference in 1956 [1]. Just after, this conference, the first Artificial Intelligence Laboratory was founded in MIT by Marvin Minsky. But the studies on Artificial Neural Networks(ANN) had also been progressing since 1940's. In 1943, McCulloch and Pitts [2] presented a mathematical model of a neuron[3].Many researchers made great contributions for improving of artificial intelligence in the following years. Rosenblat invented a learning model named Perceptron in 1958 [4]. Rosenblat implemented perceptron in MARK-1 computer for visual pattern classifier [5]. After it had been understood that perceptron could only solve linearly seperable problems (XOR curse), the interest for ANN was decreased. Marvin Minsky and Seymour Papert had shown how limited Rosenblatt's perceptron was and, how it was impossible for perceptron to learn the simple logical XOR function [6].

In 1986, Rumelhart and his friends offered a training algorithm which propagating the error [7].With backpropagation algorithm, the interest on ANNs increased again. Different type of network architectures were offered and implemented successfully such as Self Organizing Maps (SOM) by Kohonen [8], Adaptive Resonance Theory Networks [9], and Recurrent Networks [10]. But some complex classification problems weren't solved with the stated ANNs. So, deeper networks were designed, in order to solve more complex problems. But feature extraction was also another issue for scientists. ANNs classification accuracy was depend on the features presented as input. In order to overcome feature extraction, a new network model had been offered for classification of the images. In 1994, Convolutional Neural Networks [11] were used for recognition but was not studied a lot since because of the computation limits. With the increasing power of the computers, later 2010, CNN has been used for classification problems and since 2015 it reached the human classification accuracy in The ImageNet Large Scale Visual Recognition Challenge (ILSCVR) [12].

Deep learning has also been a key term in recent years with the CNNs. It has attracted much attention in computer vision and machine learning due to its high performance. Deep learning identifies the features directly from the raw data [13]. So since the feature extraction is made automatically, it fastens and makes easier to the visual tasks such as image classification. But not only classification, but also generative networks [14] were also designed and applied successfully. Especially for five years, researchers try to reveal the capabilities of computer on creativity. Images, Lyrics, Poems, scenarios have been created with recent deep learning algorithms.

Creative music with Deep Learning has been studied by a lot of researchers. The real aim of these studies are not compete with the best classics, but to create melodies for routine applications like background music of video games, since they are cheaper. DeepBach is among the studies [15]. In this study, after the network is trained on chorale harmonizations by Johann Sebastian Bach, model is capable of generating highly convincing chorales in the style of Bach. There are also softwares generating Royalty-Free musics for film and video game industry. Jukedeck, Melodrive and AIVA are the most popular ones. AIVA produces soundtracks based on deep learning architectures for any type of media [16].

MuseGAN is another study on creative music. The proposed model is based on GAN (Generative Adverserial Network). The model has been trained on a dataset of over one hundred thousand bars of rock music and applied them to generate piano-rolls of five tracks: bass, drums, guitar, piano and strings [17].

MidiNet creates midi melodies using convolutional generative adversarial network [18]. The WaveNet architecture based on a convolutional feedforward network without pooling layer is aimed at generating raw audio waveforms [19]. Hadjeres and Nielsen propose a system named AnticipationRNN [20] for generating melodies with unary constraints on notes generation by a recurrent network. The studies on Music creation using Deep Network can be found the review by Hadjeres at al[21]. 
Generative adversarial networks (GANs) are used widely in image generation, video generation and voice generation. GANs were introduced by Ian Goodfellow [14] and other researchers. There are two networks working simultaneously as a minimax model. While the first net (detector) discriminates images as real or fake, the second net(creator) tries to create real images from random normal distribution. The process stops detector can't discriminate images coming from the creator net as fake. GANs' potential is very high and there are a lot of successful aplications on creativity of the computers. Faces which are not belong to any existing person are generated by StyleGAN [22]. GANs aging face photographs show very impressive results [23]. In the recent study realized with GANs, face images of the persons are created using their voices [24]. DCGAN is created for colorization of the images [25].

Scenerio creation with deep learning has been realized too. Sunspring is a 2016 experimental science fiction short film entirely written by an artificial intelligence bot using neural networks. The script of the film was authored by a recurrent neural network called long short-term memory (LSTM) by an AI bot named Benjamin [26].

The rest of this paper is organized as follows. GANs and the most popular GAN architectures are introduced in section II. Section III presents the recent studies with GAN. Section IV presents the royalty free products and some issues because of GANs. And Section V concludes the study.

\section{Generative Adversarial Network}

Human beings have been designing devices that can do their own things for centuries. First, they designed machines that did their jobs, and then they designed computers that could do their own calculations. In recent years, human beings are developing soft ware that produces and learns like themselves. These studies can be gathered under the title of generative networks. The basis of generative adversarial network was laid in 2014 with a paper presented by Ian Goodfellow and his colleagues at the 28th Neural Information Processing Systems conference working[ 27].

GANs is a network structure obtained by simultaneous training of two deep learning network structures based on Nash game theory. One of the networks in GAN's structure is called Discriminative model and the other is called Generative model. The GANs networks structure corresponds to a two-player minimax model. In the network model, $G$ (generative) is trained to maximize the probability of $\mathrm{D}$ (discriminative) making a mistake. $\mathrm{D}$ and $\mathrm{G}$ are defined as multi-layer networks.

Generative Network consists of transposed convolutional layers, batch normalization layers and rectified linear unit layers. Generative network takes random noise array as input. With the layers, the network converts the random number arrays as desired size of output with maximum similarity.

Discriminative network consists of convolutional layers, batch normalization and other deep network layers according to the type of the GAN output.

The loss function for GAN, proposed in the literature can be formulated in general as given in the Towards a Deeper Understanding of Adversarial Losses [28] equation 1 and 2:

For Discriminative Network:

$$
\max E_{x \sim p_{d}}[f(D(x))]+E_{x}^{\sim} \sim p_{g}\left[g\left(D\left(x^{\sim}\right)\right]\right.
$$

For Generative Network:

$$
\min E_{x \sim p_{g}}\left[h\left(D\left(\mathrm{x}^{\sim}\right)\right)\right]
$$

Pd denotes the data distribution and pg is the model distribution defined by G(z) when $\mathrm{z} \sim \mathrm{pz}$.

The component functions $\mathrm{f}, \mathrm{g}$ and $\mathrm{h}$ used in Godfellow[14] studies are as given in Equation 3. 


$$
f(y)=-\log \left(1+e^{y}\right), g(y)=-y-\log \left(1+e^{y}\right), h(y)=-y-\log \left(1+e^{y}\right)
$$

Within the framework of the proposed adversarial networks, the generative model struggle against an opponent. The model is trained when the discriminator network (D) structure cannot detect whether the incoming data is from the generator network or from the data distribution.

The GANs network structure consists of 2 neural networks that work opposite to each other as seen in Figure 1 below.

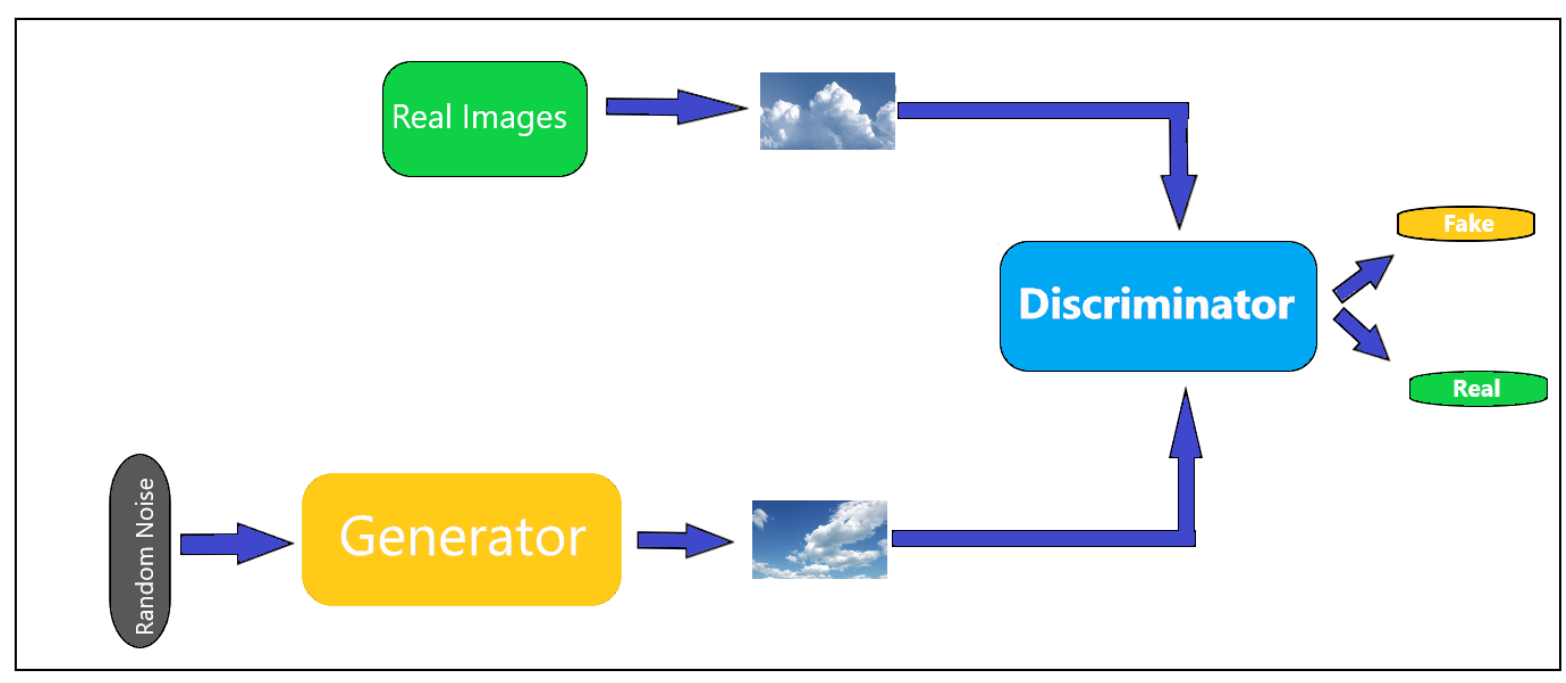

Figure 1 Generative Adversarial Network Structure

\section{Works With Generative Adversarial Network}

The studies from 2018 to today, have been searched and reviewed. The summary, results and citation numbers of the studies related to generative adversarial networks are presented in Table 1.

Recently, studies have been carried out in many areas related to GANs. In this study, studies from 2018 to date have been examined. The topics studied are in general: image restoration, resolution / super-resolution, image classification, image detection, image generation, video, image translation, image segmentation, image quality and hyperspectral image. Most of the studies reviewed are about image restoration. About image restoration; Babaee, Zhu and Sparkling (2019) presented GAN architecture to solve the problem of incomplete gait cycle. In the presented method, a generator with automatic encoder network and two discriminator are created to create full GEIs from missing GEIs. One discriminator checks whether the image is a complete GEI and the other discriminator checks whether the two GEIs belong to the same subject [29]. He and Zhang (2019) image recovery method with deep learning technique is suggested to improve underwater image recognition. The productive contention network has proven to be a perfectly suitable design to restore distorted images [30]. In the MCGAN model proposed by Wang, Fan and Zhu (2018), automatic learning of a distorted image takes place. By learning from two complementary networks, MCGAN semantically creates new pixels for the deficient areas. In comprehensive qualitative and quantitative tests on datasets, it is seen that the presented model performs better than the latest technology products [31]. In another study by Shi, $\mathrm{Li}$, and Zhu (2018), GAN architecture is proposed to automatically generate building footprints from satellite images.The results were successful when the proposed method was compared to U-Net, CGANs and other networks [32]. In the study by Gong and Zhang (2018), it was tried to solve the problem of deburring in blurry images with GAN. First, the process of dividing the fuzzy parts and clear parts of the picture is performed. Then, deburring of the fuzzy part is started. With the method created, very good results were obtained on both natural local blurred images and artificial local blurred images [33]. In this study by Zheng, Song and Wu (2019), the Encoder Directed Generative Adversarial Network (EGGAN) feature is recommended to solve the problems in face photographs. In the presented method, it is seen that GAN has reached the state of the art in terms of both quantitative 
evaluations and perceptual quality[34]. In the study by He and Zhang (2019), it is emphasized that snowflakes affect the quality of the image in pictures. In their study, they tried to solve this problem by combining generative adversarial network education model and pruning method [35]. In this study by Xiang, Wang and $\mathrm{Wu}$ (2019), a feature controlled GAN (FS-GAN) is proposed to remove raindrops from images. In the experiments, it has been observed that the presented FS-GAN outperforms state-of-the-art rain removal methods in both real world and synthetic images in terms of visual quality and quantity[36].

Considering the literature, restoration and resolution / super resolution are the most studied subjects. Studies on resoluton and super resolution; Yin (2019) presents a study on pedestrian detection techniques. The Multi-Resolution Generative Adversarial Network (MRGAN) method was used to solve the low resolution pedestrian detection problem. The presented method has yielded more successful results than previous methods [37]. Gu, Zhang, Wang (2019) propose deep DGAN to reconstruct high resolution (HR) SAR images. The experimental results also show that the proposed GAN architecture protects the GAN's SAR images with high levels of noise suppression[38]. In the article by Huang, Zhang and Zhou (2019), a new GAN design is proposed to produce SAR images over a network, improve the quality of the produced image and ultimately obtain high-resolution images. The images generated show a high similarity compared to real examples [39]. Jiang, Li and Gao (2019) propose the GAN method to analyze HSI super resolution (HSRGAN). In qualitative and quantitative results, it is seen that the presented HSRGAN performs better than technological methods such as SRGAN and SRCNN [40]. Zheng, Jiang and Zhang (2019) present a new super-resolution image reconstruction design based on SNGAN. In experiments conducted on TerraSAR and MSTAR datasets, it is seen that the proposed design performs very well in target recognition and resolution increase of SAR images [41]. Sun, Zhao and Zhang (2020), GAN architecture is presented to solve the problems of images created with SR techniques The generative model is based on a deep neural network containing a multilayer convolution module. The discriminant model consists of a multilayer neural network based on loss function. Compared to previous designs, it seems that the presented design gives better results [42]. Wang, Wu and Su (2019) use SRGAN architecture to solve single image super resolution problems in their study. Thus, they found that a clearer and more natural GAN image was produced[43]. Liu, Wang and Wan (2019) recommended CSPGAN for the synthesis of faces in their study. The experimental results show the success of CSPGAN in reconstructing photorealistic textures [44].

The next most studied topic with Gan architecture is image classification. Studies on image classification; Alnujaim, Oh and Kim (2019) use GAN architecture to classify micro-Doppler signatures measured by radar. Data has been augmented using GAN architecture to complement the data gap. The results are not conclusive, however generally show a trend. So, more research is needed to measure the quality of the GAN spectrogram [45]. Tang and Han (2019) proposed a design to define abnormal chest X-rays with GAN in their article. As a result of quantitative and qualitative experiments, it is seen that the workload is reduced for radiologists and the efficiency of the approach created and obtained 0.841 AUC in the NIH Chest X-ray data set [46]. Pan, Yu and Yi (2019) present recent studies on GANs in their article. In the first stage, the differences between productive models in recent years are examined. In the second stage, derived GAN models are classified and presented. In the third stage, training and evaluation criteria are presented. In the fourth stage, applications of GANs are presented. Finally, what studies GAN be done in the future is discussed [47]. Karadağ and Çiçek (2019) investigated the use of GAN for data enlargement in image classification. Classification performance was evaluated using three types of different methods. It is seen that GANs are used effectively for large data sets. [48]. Li, Wang and Zhang (2019) in their article, classification of urban images in SAR images is a difficult situation due to the complexity of urban areas. In order to get rid of these problems and get the proper features, this article also offers two effective solutions. Sentinel-1 SAR and GF-3 images are chosen to try the generative network. As a result of the studies, it is seen that the proposed optimization gives positive results [49]. Han, Feng and Wang (2019) proposed a new GAN in their article. With the method presented in this study, it GAN create HRRS images with a specific tag and develop image classification performance with methods based on CNN [50]. 
Considering the GAN architecture, it is seen that serious work has been done on image detection. Image detection related studies; Liu, Chen and Xu (2019) presented a semi-supervised remote sensing method based on Generative adversarial networks (GAN) in their article. Results from some high resolution remote sensing data sets also show the efficiency of the proposed design [51]. Ma, Zhou and Wang (2019) recommend using GANs to detect ships that are difficult to detect in their article. As a result of this work, the generative network, called the ship-finding network, correctly identifies difficult-to-find ships[52]. Shen, Liu and Sun (2019) propose a CNN-based detection design called LD-CNN in their article. The detection algorithm signifiGANtly decrease the calculation costs of the presented design and increases its accuracy. Also, GAN was developed to solve the problem of incomplete training and GAN effectively produced the so-called MC-GAN samples. Detection performance of the presented design; analyzed in the collected dataset and in the Munich dataset. The results of the study show that the method proposed in the Munich dataset 86.9\% (mean sensitivity) in mAP, the F1 score was 0.875 and the detection time in Nvidia Titan XP was 1.64s.When this study was done, it ensured that the results reached the most advanced level in vehicle detection [53]. Wang, Hou and Shao (2019) obtained an anomaly detection framework that provides high accuracy for electrocardiogram (ECG) signals. The proposed framework includes two models: classification model and data magnification model. The data magnification model is supported by ACGAN (auxiliary classifier). ACGAN is created by adding multiple 1-dimensional convolutional layers to the Discriminator and Generator. In experimental studies, the model was applied for sequential heartbeat detection and single-heartbeat detection[54]. García, Laparra and Chova (2019) present the use of a GAN framework to generate new satellite data in their paper. The proposed GAN method has been used for cloud detection in Landsat-8 images and Proba-V. The results show that the accuracy of GANs in detecting signifiGANtly improved[55]. Mandal, Puhan and Verma (2018) study in their article to make classification of food images more effective by using deep convolutional GAN. Considering the results of the study, the superiority of the GAN presented can be seen when looking at current approaches [56].Wadhwa, Maharana and Shah (2019) presented a draft model for facial recognition in their article. The system adopts a multi-layered approach. The system also combines draft image creation and facial recognition methods. Firstly, the transition to photo generation is made using the cGAN based pix2pix model. Then One Shot Learning is done using FaceNet. The draft recognition design developed with this study gives positive results in multiple data sets. The distinction in recognition correctness compared to the original images is seen as three percent [57].

In recent years, studies on image generation have also been observed due to the need and requirement of data sets. Studies on image generation; Gu, Zhang and Zhang (2019) present a GAN to improve the quality and accuracy of maps and aerial images conversion results in their study. Experiments on the dataset created from aerial images to the map show that the method created improves both visual appearance and accuracy by performing better than the current method [58]. Zhu, Lu and Chiang (2019) aims to create negative examples by GAN to attack facial recognition models by applying makeup effects on facial images. In the experimental results, it is seen that the method created according to previous studies creates high quality facial makeup images [59]. Chen, Zhu and Wu (2019) used GAN to expand small-sized infrared datasets in their study. In the proposed method, infrared images are created and RGB images are transformed into infrared images. Experimental results show that such an approach is efficient in enlarging the infrared dataset [60]. Chen, Yisheng, and Wang (2019) propose a new approach to increase traffic data using GANs and parallel data in their study. GANs have been applied to create artificial traffic data in parallel data method. Experimental results show that the presented design GAN increase the production of traffic data [61]. Chen, Zhang, Li (2019) aims to produce youth and old age facial image data of the same person in their study. This process is also offered using generative adversarial networks. The proposed method was applied in CADA-VS and FGNET datasets, resulting in 96.99\% -85.04\% results [62].

With Gan architecture, only pictures are not processed. Studies on text and video are also carried out. Studies on video; since 2016, various types of GAN models have been offered to provide deep learning in image coloring, image style change and image style transfer. Based on this situation, Cui and Wang (2019) used the GAN model to automatically process old documentary films [63]. The presence of haze signifiGANtly reduces visual quality and therefore video analysis negatively affects 
human-machine interaction and visual surveillance performance. Based on this situation, Pang, Xie and Li (2018) proposed a GAN to eliminate blurring in a visual signal, called HRGAN. Experimental results in databases of both synthetic and real samples show that HRGAN performs better than the most advanced algorithms in terms of efficiency [64]. Xiang, Xu and Yan (2019) propose a new GAN-based approach to hide video errors. Hiding errors in low resolution videos has been successful in both quantitative and qualitative experiments [65].

There are also studies on image translation Studies on this subject; The cloud cleaning method is an important step to improve the quality of images in general. Recently, cGAN has taken important steps in translating image to image. Based on this, Xu and Wang (2019) proposes a new objective function that increases the structural similarity rate based on cGAN. In experimental results, it shows that the presented design has a visual effect on both PSNR and cloud-covered remote sensing images [66]. Lata, Dave, and Nishanth (2019) use Conditional GANs to translate images according to some conditions. The performance of the created model is analyzed by hyper parameter adjustment [67]. Yanagi, Togo and Ogawa (2019) present a GAN-based method that converts text-to-image, which has recently become one of the popular research topics. A new "Query GAN" framework is proposed, which is based on the text-image GAN architecture. The idea presented is to use text-image images created by GAN as queries for the stage. The efficiency of the presented approach GAN be seen by experimental evaluation in which the scene is taken from the real video data sets [68].

In GAN architecture, image segmentation is important and there are a few studies on this subject. Studies on image segmentation; Gao, Peng and Li (2019) proposed road crack segmentation method based on GAN.In GAN networks, there are two neural networks, U-Net based FU-Net and CU-Net, consisting of a discriminator and a generator. While using U-Net, FU-Net and CU-Net generators, two classes of networks are used as discriminator. The three datasets provide better performance of the proposed method compared to other methods. Especially; recall, F1 score, and precision are $73.40 \%$, $77.33 \%$ and $91.46 \%$ in general data sets, respectively [69]. Skin lesion is generally considered to be a disease detected by dermoscopy images. The main goal of skin lesion analysis is to accurately segment the lesion regions. Jiang, Zhou and Qin (2019) presented a skin lesion segmentation framework based on GAN in their article. In this context, ISIC skin lesion was trained and evaluated using data sets in 2017. It shows that the presented network has obtained appropriate segmentation performance compared to other CNN approaches in the experiments [70]. Liu, Zhang and Chen (2019) investigated the data enlargement method to balance semantic tag distribution for better segmentation performance. To develop the performance of semantic segmentation networks, it has been proposed to use GAN to produce more realistic images. Looking at the results, it shows that the presented approach not only improves low accuracy segmentation performance, but an improvement of $1.3 \%$ to $2.1 \%$ in average segmentation precision. Thus, it GAN be seen that the magnification approach GAN increase accuracy and easily be applied to other segmentation approaches [71].

There are studies to improve image quality. Studies on this subject; Cao, Jia and Chen (2018) examined current GAN models and their effectiveness in computer vision applications in their article. The development and history of generative algorithms, basic network structures, GAN mechanism and analysis of the original GAN are examined. In addition, some applications of GAN in computerized images, including image translation, high quality sample production, have been explored. Then, some issues of GAN are discussed and future issues are suggested [72]. Translucent materials such as glass cause reflection problems in image processing, which reduces image quality. Li and Lun (2019) presented a new deep learning approach to eliminate reflection in their article. The image is sent to Wasserstein (WGAN) to find the background edges. Then; background edges are used in other Wasserstein GAN to recreate background images. In the results made in the study, it is seen that the method GAN reach the most advanced performance. In addition, it is seen that it is ahead of existing approaches due to the use of deep learning approaches [73]. Ultrasound has become a widely used imaging method in medicine in recent years and is applied in rural medicine, telemedicine, and community medicine. For this reason, The improvement of movable ultrasound devices has been an important issue lately. Also, the small size of movable ultrasound devices often reduce image quality. Zhou, Wang and Guo (2019) propose a new GAN model to match high-quality images corresponding to low-quality ultrasound images to overcome hardware limitations and improve image quality of 
Özkan et. al

mobile ultrasound devices. In the results obtained in the study, it shows that the presented method gives optimum results to improve quality and movable ultrasound images[74].

Studies on hyperspectral image; Förster, Behley and Behmann (2019) use Cycle-Consistent GAN to predict and prevent the spread of disease symptoms to barley plants. In the experiments carried out within the scope of the study, it GAN be seen that the model GAN learn the spread of the disease, which is shown both visually and in the relevant reflection spectra [75]. Although HSI Classification has been researched for the past decade, the challenges remain due to the limited number of labeled samples. Wang, Tao and Qi (2019) semi-supervised variable GAN is proposed to overcome this difficulty in their article. In the proposed model, when the number of labeled data is low, HSI classification performs better than conditional GAN [76]. As you can see, GANs are used in many different areas and for different purposes. Studies on GANs are not limited to these and will become more popular over time. The main topics in this study on GANs and how many studies have been examined on that subject are shown in Table 1.

Table 1 Main Categories Reviewed

\begin{tabular}{|c|c|}
\hline The categories & The number of studies \\
\hline Image restoration & 8 \\
\hline Resoluiton /Super-resolution & 8 \\
\hline Image detection & 7 \\
\hline Image classification & 6 \\
\hline Images generation & 5 \\
\hline Video analyze & 3 \\
\hline Image translation & 3 \\
\hline Image segmentation & 3 \\
\hline Image guality & 3 \\
\hline Hyperspectral image & 2 \\
\hline
\end{tabular}

When the latest studies on GAN networks are examined, it is seen in Table 1 that the most studied topics are image restoration, resoluiton / super-resolution and image detection. These three studies are followed by image classification, images generation, video analyze, image translation, image segmentation. Additionally, image guality and hyperspectral image are the least studied topics.

The status of the examined 48 articles according to the number of citations is shown in the graphic in Figure 2. Citation counts are taken from Google Scholar. Since the studies are generally new, it is seen that the citation numbers of most articles are between 0 and 5 .

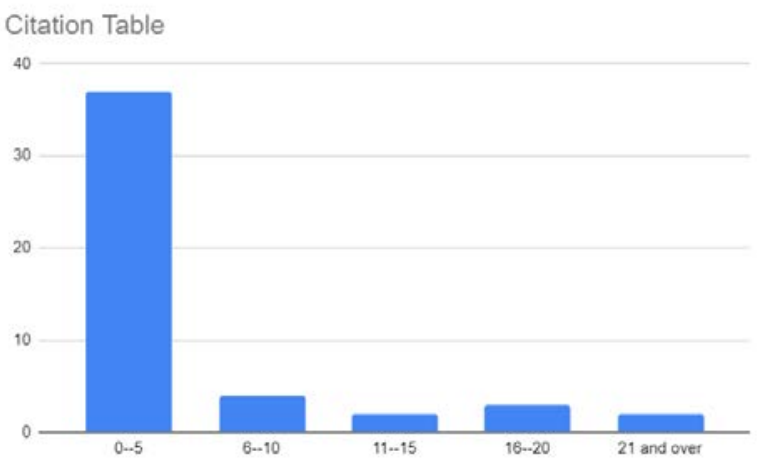

Figure 2 Citation Table

\section{Royalty-Free Products and Issues Coming with GANs}

Royalty-free (RF) material means that it can be used without the need to pay royalties or license fees for each use. If image has copyright[77] licence, the user must make payment to the licensor. In a very near future, due to GANs a vast variety of productions are created by GANs. But this production will bring copyright problem. Royalty-free music, realistic face images, paintings created by 
computers, fake voices create authentication problems. For real persons, creativity are protected by laws, but what about the computers?

If we are using some part or whole part of music, we must pay royalties. But in recent applications, Vincent Van Gogh style images or Johann Sebastian Bach style musics are created using style transfer. If such type of GANs trained with the material which requires copyright, what will be the created products? Royalty free or not.

Another issue is authentication problem. Created fake faces can be used for forgery. Or due to GANs, fake voices can be created mimicking the previous speeches. If fabric voice says "Hey Siri!" and use our personel mobile phone using fabric voices, what will be the results and regulations? So computer productivity is also an issue like Genetic Cloning. So the possible side effects, or negative effects must be discussed.

Another issue is not negative but must be considered. In a very near future, with the increasing performance of computers and GANs we will witness very successful and creative art works. Does this affect people's creativity negatively? With powerfull computers, very high resolution images can also be created using GANs. This creativity will bring two issues. One of them positive, the other one is negative. One the one hand, creativity strengthen Game Industry. Because desired scenes can easily be created using GANs. Unlimited characters can be cerated for a game. On the other hand, this creativity will decrease the need for some jobs, as in the industrial revolution. For the time being, even if computers can't discriminate the realistic images or voices, can seeing too many realistic photos, weaken people's discrimination?

When the literature studies are examined, it is seen that there is no study on royalty-free products until now. Generally, there is a gap in royalty-free products as image resolution, image restoration and image detection issues are studied. For these reasons, it is planned to work on royalty-free products in the next study.

\section{Conclusion}

As can be seen from recent studies with GANs, the potential of GAN is quite high. Discovered in 2014, GAN has increased its popularity today. Especially the production of new images, new sounds and new texts has become a very interesting issue for those who need this data.

GANs generally seem to work with image resolution, image detection and image restoration. In addition, there are studies on image quality without creating hyperspectral images. These issues have been studied less than other issues, but it is predicted that there may be serious increases in these issues. At the same time, it is seen that GAN architecture is still not used in many areas as well as the subjects studied. While doing the literature study, we tried to summarize the most effective studies published since 2018 and found that no work has been done on Royalty-free products. In this study, we tried to give a perspective for future studies by presenting a general overview of GANs.

\section{References}

[1] P. McCorduck, M. Minsky, Selfridge, O. G. \& H. A. Simon, History of Artificial Intelligence. In IJCAI , pp. 951-954,1997.

[2] W. S. McCulloch and W. Pitts, “A logical calculus of the ideas immanent in neurons activity,” Bull. Math. Biophys., vol. 5, pp. 115-133, 1943.

[3] L. Zhang and B. Zhang, "A geometrical representation of McCulloch-Pitts neural model and its applications," in IEEE Transactions on Neural Networks, vol. 10, no. 4, pp. 925-929, 1999. 
[4] F. Rosenblatt, The perceptron: A probabilistic model for information storage and organization in the brain. Psychological Review, 65(6), pp. 386-408,1958.

[5] pace, “pace homepage,” 2019. [Online]. Available:

http://csis.pace.edu/ ctappert/srd2011/rosenblatt-contributions.htm [Accessed: 20-Feb-2020].

[6] M. Minsky, \& S. Papert, An introduction to computational geometry. Cambridge tiass., HIT, 1969.

[7] D. E. Rumelhart, E. H. Geoffrey, and R. J. Williams, Learning internal representations by error propagation. No. ICS-8506. California Univ San Diego La Jolla Inst for Cognitive Science, 1985.

[8] T. Kohonen, "The self-organizing map," in Proceedings of the IEEE, vol. 78, no. 9, pp. 14641480, 1990.

[9] J. Elman, “Finding structure in time,” Cognitive Science, vol. 14, pp. 179-211, 1990.

[10] G. A.Carpenter, S. Grossberg, and D. B. Rosen. "Fuzzy ART: Fast stable learning and categorization of analog patterns by an adaptive resonance system." Neural networks 4.6, pp.759-771,1991.

[11] Y. L. Cun and Y. Bengio, "Word-level training of a handwritten word recognizer based on convolutional neural networks," Proceedings of the 12th IAPR International Conference on Pattern Recognition, Conference C: Signal Processing (Cat. No.94CH3440-5), Jerusalem, Israel, vol.2, pp. 88-92, 1994.

[12] O. Russakovsky, J. Deng, , H. Su et al., ImageNet Large Scale Visual Recognition Challenge. Int J Comput Vis 115, pp. 211-252, 2015.

[13] J. Hu, J. Lu, Y. Tan and J. Zhou, "Deep Transfer Metric Learning," in IEEE Transactions on Image Processing, vol. 25, no. 12, pp. 5576-5588, 2016.

[14] I.Goodfellow, J. Pouget-Abadie, M. Mirza, B. Xu, D. Warde-Farley, S. Ozair, \& Y. Bengio, Generative adversarial nets. In Advances in neural information processing systems, pp. 26722680,2014

[15] Hadjeres, Gaëtan, F. Pachet, and F. Nielsen, "Deepbach: a steerable model for bach chorales generation." Proceedings of the 34th International Conference on Machine Learning-Volume $70,2017$.

[16] aiva, “aiva homepage,” 2019. [Online]. Available: https://www.aiva.ai/ [Accessed: 21-Feb2020].

[17] Dong, Hao-Wen et al., "MuseGAN: Symbolic-domain music generation and accompaniment with multi-track sequential generative adversarial networks.", 2017.

[18] L. Yang, S. Chou, and Y. Yang. MidiNet: A convolutional generative adversarial network for 
symbolic-domain music generation. In Proceedings of the 18th International Society for Music Information Retrieval Conference (ISMIR 2017), Suzhou, China, 2017.

[19] A. Oord, S.Dieleman, H. Zen, K. Simonyan, O. Vinyals, A. Graves, N. Kalchbrenner, A. Senior, and K. Kavukcuoglu. WaveNet: A generative model for raw audio, 2016.

[20] Hadjeres, Gaëtan, and F. Nielsen. "Anticipation-RNN: Enforcing unary constraints in sequence generation, with application to interactive music generation." Neural Computing and Applications, pp.1-11,2018.

[21] Briot, J. Pierre, G. Hadjeres, and F. Pachet. "Deep learning techniques for music generation--a survey.", 2017.

[22] Karras, Tero, S.Laine, and T. Aila. "A style-based generator architecture for generative adversarial networks." Proceedings of the IEEE Conference on Computer Vision and Pattern Recognition. 2019.

[23] Antipov, Grigory, M. Baccouche, and J. Dugelay. "Face aging with conditional generative adversarial networks." 2017 IEEE international conference on image processing (ICIP). IEEE, 2017.

[24] Oh, T.Hyun, et al.. "Speech2face: Learning the face behind a voice." Proceedings of the IEEE Conference on Computer Vision and Pattern Recognition. 2019.

[25] Heo, Hwan, and Y. Hwang. "Automatic Sketch Colorization using DCGAN." 2018 18th International Conference on Control, Automation and Systems (ICCAS). IEEE, 2018.

[26] thereforefilms, "thereforefilms homepage,” 2016. [Online]. Available: http://www.thereforefilms.com/sunspring.html [Accessed: 21-Feb-2020].

[27] I.Goodfellow, J. Pouget-Abadie, M.Mirza, B. Xu, D.Warde-Farley, S. Ozair, \& Y. Bengio, Generative adversarial nets. In Advances in neural information processing systems ,pp. 26722680, 2014.

[28] Dong, Hao-Wen, and Y. Yang, "Towards a deeper understanding of adversarial losses.", 2019.

[29] M. Babaee, Y. Zhu, O. Köpüklü, S. Hörmann and G. Rigoll, "Gait Energy Image Restoration Using Generative Adversarial Networks," 2019 IEEE International Conference on Image Processing (ICIP), Taipei, Taiwan, pp. 2596-2600, 2019.

[30] C. He and Z. Zhang, "Restoration of Underwater Distorted Image Sequence Based on Generative Adversarial Network," 2019 IEEE 8th Joint International Information Technology and Artificial Intelligence Conference (ITAIC), Chongqing, China, pp. 866-870, 2019.

[31] Q. Wang, H. Fan, L. Zhu and Y. Tang, "Deeply Supervised Face Completion With MultiContext Generative Adversarial Network," in IEEE Signal Processing Letters, vol. 26, no. 3, pp. 400-404, 2019. 
[32] Y. Shi, Q. Li and X. X. Zhu, "Building Footprint Generation Using Improved Generative Adversarial Networks," in IEEE Geoscience and Remote Sensing Letters, vol. 16, no. 4, pp. 603-607,2019.

[33] G. Gong and K. Zhang, "Local Blurred Natural Image Restoration Based on Self-Reference Deblurring Generative Adversarial Networks," 2019 IEEE International Conference on Signal and Image Processing Applications (ICSIPA), Kuala Lumpur, Malaysia, pp. 231-235, 2019.

[34] J. Zheng, W. Song, Y. Wu, R. Xu and F. Liu, "Feature Encoder Guided Generative Adversarial Network for Face Photo-Sketch Synthesis," in IEEE Access, vol. 7, pp. 154971-154985, 2019.

[35] L. He and J. Zhang, "Snowflakes Removal for Single Image Based on Model Pruning and Generative Adversarial Network," 2019 IEEE 4th International Conference on Image, Vision and Computing (ICIVC), Xiamen, China, pp. 172-176, 2019.

[36] P. Xiang, L. Wang, F. Wu, J. Cheng and M. Zhou, "Single-Image De-Raining With FeatureSupervised Generative Adversarial Network," in IEEE Signal Processing Letters, vol. 26, no. 5, pp. 650-654, 2019.

[37] R. Yin, "Multi-Resolution Generative Adversarial Networks for Tiny-Scale Pedestrian Detection," 2019 IEEE International Conference on Image Processing (ICIP), Taipei, Taiwan, pp. 1665-1669, 2019.

[38] F. Gu, H. Zhang, C. Wang and F. Wu, "SAR Image Super-Resolution Based on Noise-Free Generative Adversarial Network," IGARSS 2019 - 2019 IEEE International Geoscience and Remote Sensing Symposium, Yokohama, Japan, pp. 2575-2578, 2019.

[39] H. Huang, F. Zhang, Y. Zhou, Q. Yin and W. Hu, "High Resolution SAR Image Synthesis with Hierarchical Generative Adversarial Networks," IGARSS 2019 - 2019 IEEE International Geoscience and Remote Sensing Symposium, Yokohama, Japan, pp. 2782-2785, 2019.

[40] R. Jiang et al., "Learning Spectral and Spatial Features Based on Generative Adversarial Network for Hyperspectral Image Super-Resolution," IGARSS 2019 - 2019 IEEE International Geoscience and Remote Sensing Symposium, Yokohama, Japan, pp. 3161-3164, 2019.

[41] C. Zheng, X. Jiang, Y. Zhang, X. Liu, B. Yuan and Z. Li, "Self-Normalizing Generative Adversarial Network for Super-Resolution Reconstruction of SAR Images," IGARSS 2019 2019 IEEE International Geoscience and Remote Sensing Symposium, Yokohama, Japan, pp. 1911-1914, 2019.

[42] H. Liu, F. Wang and L. Liu, "Image Super-resolution Reconstruction Based on an Improved Generative Adversarial Network," 2019 1st International Conference on Industrial Artificial Intelligence (IAI), Shenyang, China, pp. 1-6, 2019.

[43] H. Wang, W. Wu, Y. Su, Y. Duan and P. Wang, "Image Super-Resolution using a Improved Generative Adversarial Network," 2019 IEEE 9th International Conference on Electronics Information and Emergency Communication (ICEIEC), Beijing, China, pp. 312-315, 2019.

[44] L. Liu, S. Wang and L. Wan, "Component Semantic Prior Guided Generative Adversarial 
Network for Face Super-Resolution," in IEEE Access, vol. 7, pp. 77027-77036, 2019.

[45] I. Alnujaim, D. Oh and Y. Kim, "Generative Adversarial Networks to Augment Micro-Doppler Signatures for the Classification of Human Activity," IGARSS 2019 - 2019 IEEE International Geoscience and Remote Sensing Symposium, Yokohama, Japan, pp. 9459-9461, 2019.

[46] Y. Tang, Y. Tang, M. Han, J. Xiao and R. M. Summers, "Abnormal Chest X-Ray Identification With Generative Adversarial One-Class Classifier," 2019 IEEE 16th International Symposium on Biomedical Imaging (ISBI 2019), Venice, Italy, pp. 1358-1361, 2019.

[47] Z. Pan, W. Yu, X. Yi, A. Khan, F. Yuan and Y. Zheng, "Recent Progress on Generative Adversarial Networks (GANs): A Survey," in IEEE Access, vol. 7, pp. 36322-36333, 2019.

[48] Ö. Ö. Karadağ and Ö. Erdaş Çiçek, "Experimental Assessment of the Performance of Data Augmentation with Generative Adversarial Networks in the Image Classification Problem," 2019 Innovations in Intelligent Systems and Applications Conference (ASYU), Izmir, Turkey, pp. 1-4, 2019.

[49] L. Li, C. Wang, H. Zhang and K. Zhang, "SAR Image Urban Scene Classification based on an Optimized Conditional Generative Adversarial Network," 2019 SAR in Big Data Era (BIGSARDATA), Beijing, China, pp. 1-4, 2019.

[50] W. Han, R. Feng, L. Wang and J. Chen, "Supervised Generative Adversarial Network Based Sample Generation for Scene Classification," IGARSS 2019 - 2019 IEEE International Geoscience and Remote Sensing Symposium, Yokohama, Japan, pp. 3041-3044, 2019.

[51] J. Liu et al., "Semi-Supervised Change Detection Based on Graphs with Generative Adversarial Networks," IGARSS 2019 - 2019 IEEE International Geoscience and Remote Sensing Symposium, Yokohama, Japan, pp. 74-77, 2019.

[52] J. Ma, Z. Zhou, B. Wang and Z. An, "Hard Ship Detection via Generative Adversarial Networks," 2019 Chinese Control And Decision Conference (CCDC), Nanchang, China, pp. 3961-3965, 2019.

[53] J. Shen, N. Liu, H. Sun and H. Zhou, "Vehicle Detection in Aerial Images Based on Lightweight Deep Convolutional Network and Generative Adversarial Network," in IEEE Access, vol. 7, pp. 148119-148130, 2019.

[54] P. Wang, B. Hou, S. Shao and R. Yan, "ECG Arrhythmias Detection Using Auxiliary Classifier Generative Adversarial Network and Residual Network," in IEEE Access, vol. 7, pp. 100910-100922, 2019.

[55] G. Mateo-García, V. Laparra and L. Gómez-Chova, "Domain Adaptation of Landsat-8 and Proba-V Data Using Generative Adversarial Networks for Cloud Detection," IGARSS 2019 2019 IEEE International Geoscience and Remote Sensing Symposium, Yokohama, Japan, pp. 712-715, 2019.

[56] B. Mandal, N. B. Puhan and A. Verma, "Deep Convolutional Generative Adversarial NetworkBased Food Recognition Using Partially Labeled Data," in IEEE Sensors Letters, vol. 3, no. 2, 
pp. 1-4, 2019.

[57] D. Wadhwa, U. Maharana, D. Shah, V. Yadav and P. Pandey, "Human Sketch Recognition using Generative Adversarial Networks and One-Shot Learning," 2019 Twelfth International Conference on Contemporary Computing (IC3), Noida, India, pp. 1-6, 2019.

[58] J. Gu et al., "Aerial Image and Map Synthesis Using Generative Adversarial Networks," IGARSS 2019 - 2019 IEEE International Geoscience and Remote Sensing Symposium, Yokohama, Japan, pp. 9803-9806, 2019.

[59] Z. Zhu, Y. Lu and C. Chiang, "Generating Adversarial Examples By Makeup Attacks on Face Recognition," 2019 IEEE International Conference on Image Processing (ICIP), Taipei, Taiwan, pp. 2516-2520, 2019.

[60] F. Chen, F. Zhu, Q. Wu, Y. Hao, Y. Cui and E. Wang, "InfraRed Images Augmentation Based on Images Generation with Generative Adversarial Networks," 2019 IEEE International Conference on Unmanned Systems (ICUS), Beijing, China, pp. 62-66. 2019.

[61] Y. Chen, Y. Lv and F. Wang, "Traffic Flow Imputation Using Parallel Data and Generative Adversarial Networks," in IEEE Transactions on Intelligent Transportation Systems, vol. 21, no. 4, pp. 1624-1630, 2020.

[62] S. Chen, D. Zhang, L. Yang and P. Chen, "Age-invariant Face Recognition Based on Sample Enhancement of Generative Adversarial Networks," 2019 6th International Conference on Systems and Informatics (ICSAI), Shanghai, China, pp. 388-392, 2019.

[63] Y. Cui and W. Wang, "Colorless Video Rendering System via Generative Adversarial Networks," 2019 IEEE International Conference on Artificial Intelligence and Computer Applications (ICAICA), Dalian, China, pp. 464-467, 2019.

[64] Y. Pang, J. Xie and X. Li, "Visual Haze Removal by a Unified Generative Adversarial Network," in IEEE Transactions on Circuits and Systems for Video Technology, vol. 29, no. 11, pp. 3211-3221, 2019.

[65] C. Xiang, J. Xu, C. Yan, Q. Peng and X. Wu, "Generative Adversarial Networks Based Error Concealment for Low Resolution Video," ICASSP 2019 - 2019 IEEE International Conference on Acoustics, Speech and Signal Processing (ICASSP), Brighton, United Kingdom, pp. 18271831,2019 .

[66] X. Wang, G. Xu, Y. Wang, D. Lin, P. Li and X. Lin, "Thin and Thick Cloud Removal on Remote Sensing Image by Conditional Generative Adversarial Network," IGARSS 2019 - 2019 IEEE International Geoscience and Remote Sensing Symposium, Yokohama, Japan, pp. 14261429, 2019.

[67] K. Lata, M. Dave and K. N. Nishanth, "Image-to-Image Translation Using Generative Adversarial Network," 2019 3rd International conference on Electronics, Communication and Aerospace Technology (ICECA), Coimbatore, India, 2019, pp. 186-189.

[68] R. Yanagi, R. Togo, T. Ogawa and M. Haseyama, "Query is GAN: Scene Retrieval With 
Attentional Text-to-Image Generative Adversarial Network," in IEEE Access, vol. 7, pp. 153183-153193, 2019.

[69] Z. Gao, B. Peng, T. Li and C. Gou, "Generative Adversarial Networks for Road Crack Image Segmentation," 2019 International Joint Conference on Neural Networks (IJCNN), Budapest, Hungary, pp. 1-8, 2019.

[70] F. Jiang, F. Zhou, J. Qin, T. Wang and B. Lei, "Decision-Augmented Generative Adversarial Network for Skin Lesion Segmentation," 2019 IEEE 16th International Symposium on Biomedical Imaging (ISBI 2019), Venice, Italy, pp. 447-450, 2019.

[71] S. Liu, J. Zhang, Y. Chen, Y. Liu, Z. Qin and T. Wan, "Pixel Level Data Augmentation for Semantic Image Segmentation Using Generative Adversarial Networks," ICASSP 2019 - 2019 IEEE International Conference on Acoustics, Speech and Signal Processing (ICASSP), Brighton, United Kingdom, pp. 1902-1906, 2019.

[72] Y. Cao et al., "Recent Advances of Generative Adversarial Networks in Computer Vision," in IEEE Access, vol. 7, pp. 14985-15006, 2019.

[73] T. Li and D. P. K. Lun, "Image Reflection Removal Using the Wasserstein Generative Adversarial Network," ICASSP 2019 - 2019 IEEE International Conference on Acoustics, Speech and Signal Processing (ICASSP), Brighton, United Kingdom, pp. 1-5, 2019.

[74] Z. Zhou, Y. Wang, Y. Guo, Y. Qi and J. Yu, "Image Quality Improvement of Hand-Held Ultrasound Devices With a Two-Stage Generative Adversarial Network," in IEEE Transactions on Biomedical Engineering, vol. 67, pp. 298-311, 2020.

[75] A. Förster, J. Behley, J. Behmann and R. Roscher, "Hyperspectral Plant Disease Forecasting Using Generative Adversarial Networks," IGARSS 2019 - 2019 IEEE International Geoscience and Remote Sensing Symposium, Yokohama, Japan, pp. 1793-1796, 2019.

[76] H. Wang, C. Tao, J. Qi, H. Li and Y. Tang, "Semi-Supervised Variational Generative Adversarial Networks for Hyperspectral Image Classification," IGARSS 2019 - 2019 IEEE International Geoscience and Remote Sensing Symposium, Yokohama, Japan, pp. 9792-9794, 2019.

[77] copyright, “copyright homepage,” 2020. [Online]. Available: https://www.copyright.gov/ [Accessed: 21-Apr-2020].

\section{Biography}

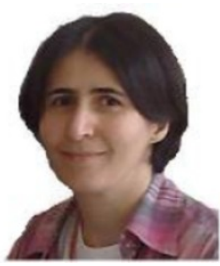

ERDOGMUS P. was born in Erzurum in 1972. She received the B.S. in Electronic and Communication Engineering from Yildiz Technical University, Kocaeli Engineering Faculty in 1993, and M.S. and Ph.D degree Computer Sciences and Numerical Methods from Ataturk University in 1997, 2003 respectively. From 2003 to 2010, she was Assistant Professor in Duzce University, Technical Education Faculty. From 2013 to 2019 she has worked in Computer Engineering Department of Duzce University, Engineering Faculty as an associate professor. She is working as professor now. Her research interests are machine learning, signal and image processing, nature-inspired optimization algorithms, deep learning. 
Özkan et. al

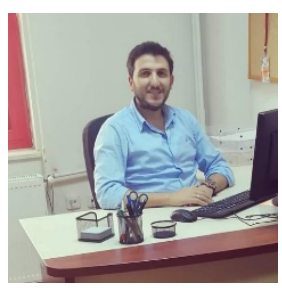

OZKAN Y. I was born in Eskişehir in 1990.I completed my undergraduate education in Computer Education and Educational Technology at Hacettepe University between 2009-2013. I completed my master's degree in the Department of Electrical Electronics and Computer Engineering at Düzce University. Now, I continue to the same department at Düzce University and I am a PhD. I work as a teaching assistant at Zonguldak Bülent Ecevit University Kdz. Ereğli Vocational School. 